\title{
ERRATUM
}

Jens Tschmelak · Guenther Proll · Guenter Gauglitz

\section{Ultra-sensitive fully automated immunoassay for detection of propanil in aqueous samples: steps of progress toward sub-nanogram per liter detection}

Published online: 18 December 2004

(C) Springer-Verlag 2004

\section{Anal Bioanal Chem (2004) 379:1004-1012}

Unfortunately, there were errors in the units given in Tables 1 and 3 and wrong numbers for signal, noise, and

Table 1 Summary of results from comparison of surface modification with all parameters of the logistic fit functions $\left(A_{1}, A_{2}, x_{0}\right.$, and $p$ ) and the validation parameters (LOD and LOQ) for all four calibration curves (comment: the parameter $x_{0}$ of the logistic fit function is equivalent to the test-mid point $\mathrm{IC}_{50}$ )

\begin{tabular}{|c|c|c|c|c|}
\hline Comparison & No. 1 & No. 2 & No. 3 & No. 4 \\
\hline Surface & Propanil-h4 & Propanil-h5 & Propanil-itc & Propanil-hg \\
\hline$A_{1}(\%)$ & 100.00 & 100.00 & 100.00 & 100.00 \\
\hline$A_{2}(\%)$ & 32.14 & 11.00 & 5.40 & 11.76 \\
\hline$x_{0}\left(\mu \mathrm{g} \mathrm{L}^{-1}\right)$ & 0.25 & 0.19 & 0.43 & 0.42 \\
\hline$p$ & 0.98 & 1.02 & 0.85 & 0.95 \\
\hline$c_{\mathrm{ab}}\left(\mathrm{ng} \mathrm{mL}^{-1}\right)$ & 200.0 & 200.0 & 200.0 & 200.0 \\
\hline $\operatorname{LOD}\left(\mathrm{ng} \mathrm{L}^{-1}\right)$ & 7.9 & 13.5 & 13.4 & 7.6 \\
\hline LOQ (ng $\mathrm{L}^{-1}$ ) & 29.4 & 52.2 & 65.1 & 28.3 \\
\hline
\end{tabular}

SNR in case of "Trial 6" in Table 3 of this article. The corrected versions are given below.

Table 3 Summary of the validation parameters $\left(\mathrm{IC}_{50}, \mathrm{SD}_{\mathrm{zs}}, \mathrm{LOD}, \mathrm{LOQ}\right.$, signal, noise, SNR, SD-range) for all six calibration curves with the different antibody concentrations $\left(c_{\mathrm{AB}}\right)$ between 3.1 and $100.0 \mathrm{ng} \mathrm{mL} \mathrm{m}^{-1}$ a-PRN

\begin{tabular}{|c|c|c|c|c|c|c|}
\hline Validation parameters & Trial 1 & Trial 2 & Trial 3 & Trial 4 & Trial 5 & Trial 6 \\
\hline$c_{\mathrm{ab}}\left(\mathrm{ng} \mathrm{mL}^{-1}\right)$ & 100.0 & 50.0 & 25.0 & 12.5 & 6.3 & 3.1 \\
\hline $\mathrm{IC}_{50}\left(\mathrm{ng} \mathrm{L^{-1 }}\right)$ & 145 & 100 & 85 & 62 & 46 & 52 \\
\hline $\mathrm{SD}_{\mathrm{zs}}(\%)$ & 1.58 & 1.25 & 0.81 & 1.91 & 1.30 & 1.29 \\
\hline $\operatorname{LOD}\left(n g L^{-1}\right)$ & 7.8 & 3.2 & 1.2 & 1.5 & 0.8 & 0.6 \\
\hline LOQ (ng L L $\left.L^{-1}\right)$ & 29.9 & 13.6 & 5.4 & 10.3 & 5.2 & 4.5 \\
\hline Signal (mean value) $(\mu \mathrm{V})$ & 71.49 & 36.69 & 23.21 & 10.53 & 5.09 & 2.71 \\
\hline Noise (mean value) $(\mu \mathrm{V})$ & 0.11 & 0.13 & 0.12 & 0.11 & 0.11 & 0.11 \\
\hline SNR & 676 & 292 & 186 & 96 & 45 & 25 \\
\hline SD-range $(\%)$ & $0.63-3.21$ & $0.17-2.50$ & $0.65-2.03$ & $0.52-3.51$ & $0.48-4.67$ & $1.15-2.87$ \\
\hline
\end{tabular}

J. Tschmelak $(\varangle) \cdot$ G. Proll · G. Gauglitz Institute of Physical and Theoretical Chemistry (IPTC),

Eberhard-Karls-University of Tuebingen,

Auf der Morgenstelle 8, 72076 Tuebingen, Germany

Tel.: + 49-7071-2974668

Fax: + 49-7071-295490

E-mail: jens.tschmelak@ipc.uni-tuebingen.de http://barolo.ipc.uni-tuebingen.de 